\title{
Content and Acceptability of an Occupational Therapy Intervention in Homecare Re-ablement Services (OTHERS)
}

\author{
Occupational Therapy Intervention in Reablement
}

1. Phillip J Whitehead, Associate Professor - Occupational Therapy, Department of Social Work, Education and Community Wellbeing, Northumbria University, UK

2. Avril ER Drummond, Professor of Healthcare Research, Faculty of Medicine and Health Sciences, University of Nottingham, UK

3. Ruth H Parry, Professor of Human Communication and Interaction, Department of Social Sciences, Loughborough University, UK

4. Marion F Walker, Professor of Stroke Rehabilitation and Associate Pro-Vice Chancellor (Equality and Diversity), School of Medicine, University of Nottingham, UK

Corresponding author: Dr Phillip Whitehead, Associate Professor - Occupational Therapy, Department of Social Work, Education and Community Wellbeing, Northumbria University, Coach Lane Campus, Benton, Newcastle-Upon-Tyne, NE7 7XA, UK.

phillip.whitehead@northumbria.ac.uk

\begin{abstract}
Introduction: Despite clear compatibilities between the tenets of occupational therapy and re-ablement, there is limited research on occupational therapy in homecare reablement services. This paper describes the content of an occupational therapy intervention that was delivered in homecare re-ablement services as part of a feasibility randomised controlled trial (OTHERS). It also evaluates whether the intervention was acceptable to the participants who received it.
\end{abstract}

Method: There were three phases. 1.) A bespoke pro forma was completed recording the activities undertaken after each therapy visit. 2.) An acceptability questionnaire was sent by post to every intervention participant. 3.) Semi-structured interviews were completed with key informants who received the intervention.

Results/Findings: The principal activities undertaken were: assessment; case management; goal setting; advice and support; and practising activities. It was possible to implement a graded programme for participants' main goals in relation to bathing/showering or kitchen activities. Participants particularly valued the advice and support provided, however, there were difficulties due to fluctuations in circumstances and with activities of daily living ( $A D L$ ) outside the home. Participants also had outdoor mobility goals but there were difficulties working on these within the 6-weeek timescale.

Conclusion: An intervention focussing on ADL within the home was acceptable for participants and consistent with their goals and objectives; however, they also had goals beyond personal ADL and the timescale of the re-ablement episode which were not met. Further research should focus on extended ADL at a later stage beyond the time-limited period.

Key Words: Re-ablement; Homecare; ADL; Acceptability of Intervention 


\section{Introduction}

Re-ablement has been identified as one of the 'top ten' prevention interventions for older adults (Allen and Glasby, 2013) and is highlighted within the statutory guidance to support The Care Act 2014 as an example of a tertiary prevention service (Department of Health, 2017). Although the College of Occupational Therapists (2010) argued that the involvement of occupational therapists in re-ablement services is essential to successful outcomes for service users and service providers, there is a dearth of evidence regarding the effectiveness of re-ablement in general (Aspinal et al., 2016) and the specific roles of individual professionals and staff groups within re-ablement services (Pettersson and Iwarsson, 2017). The Social Care Institute for Excellence (2011) stated that comparing the effectiveness and cost effectiveness of re-ablement teams employing occupational therapists with those that do not was a research priority. In order to evaluate effectiveness, we conducted a feasibility randomised controlled trial (RCT) of Occupational Therapy in Homecare Re-ablement Services (OTHERS) (Whitehead et al., 2014). OTHERS aimed to determine the feasibility of randomising re-ablement service users to receive an occupational therapy intervention or to usual care, which did not involve routine occupational therapy input. The results showed improvements from baseline in both groups across a range of activities of daily living and quality of life outcomes, although overall trends were stronger in the occupational therapy intervention group. We concluded that the RCT was feasible, subject to certain caveats and these results are reported elsewhere (Whitehead et al., 2016).

Feasibility studies are essential for the future evidence base underpinning occupational therapy; an essential component of such studies is to determine the practicalities of delivering an intervention in the context of a research study (Drummond, 2017). Guidelines from the Medical Research Council highlight the importance of providing a clear description of the intervention alongside the processes involved in delivering it (Craig et al., 2013). Thus, reports of randomised controlled trials may be criticised if they provide incomplete descriptions of interventions (Walker et al., 2017). Insufficient description can cause difficulties with replicating that intervention in clinical practice or in further research (Wilkins et al., 2003; Hoffmann et al., 2014; Hasson et al., 2012). Furthermore, in a feasibility study it is important to evaluate whether the intervention itself was viable and practical, such that it could be delivered as intended. It is also important to investigate whether the participants in the study found the intervention to be acceptable. Problems with acceptability often undermine evaluations of interventions (Craig et al., 2013) because the participants may not adhere. It is therefore important to specifically examine acceptability when reporting the findings from a randomised controlled trial. 
The National Audit for Intermediate Care defines re-ablement as being predominantly delivered by social care professionals (NHS England, 2014). It is commonly linked with homecare services and often staffed by former homecare workers who are urged to 'standback' and encourage the user to carry out tasks independently wherever possible (Le Mesurier and Cumella, 1999). The OTHERS trial was conducted within a defined geographical patch of a single-site local authority homecare re-ablement team. The service did not routinely provide input from therapists and was staffed primarily by social care reablement workers. For OTHERS, inclusion criterion was the ability to provide informed written consent. Exclusion criteria were inability to speak English, on an end-of-life care pathway, requiring assistance from two or more people to assist with a transfer, or receiving input from another community rehabilitation team (such as the community stroke team).

The aim of this paper is to provide a detailed description of the content of the occupational therapy intervention that was provided in OTHERS and to evaluate whether the intervention was acceptable to the participants who received it.

\section{Method}

There were three phases to this evaluation of intervention content and acceptability: 1 . The activities undertaken by the occupational therapist were recorded using a bespoke pro forma. 2. An acceptability questionnaire was sent to each participant who received the intervention 3. Qualitative interviews were completed with key-informants who received the intervention. Ethical approval was obtained from the Social Care Research Ethics Committee (Ref: 13/IEC08/002). Data collection was completed in 2014.

\section{Recording and Analysing the Content of the Intervention}

In order to describe the content of the intervention, a bespoke pro forma was designed to capture and record the visit content and actions undertaken by the occupational therapist. The pro forma was designed with reference to previous studies describing occupational therapists' interventions within evaluative studies (Grant et al., 2014; Phillips et al., 2010). It covered the following areas: the length of time spent on the visit, the goals set and goals reviewed (achieved, partially achieved, not achieved), and any equipment or minor adaptations provided. Free text notes on the visits were also completed. Each visit was categorised (in five minute intervals) into time spent on the following activities listed as key intervention components in the published protocol:

- assessment

- goal setting

- goal reviewing 
- teaching techniques

- practising activities

- providing advice or generic 'case management'.

The focus of the intervention was on activities of daily living (ADL) within the home and this was based on the findings of previous work (Whitehead et al., 2015; Whitehead, 2016). In addition, the overall visit was categorised into the proportion of time spent on particular ADL:

- indoor mobility

- transfers

- bathing or showering

- strip washing

- dressing

- kitchen activities

- stair mobility

- outdoor mobility

- toileting

- access

- any other activities.

The visit pro forma was completed electronically by the occupational therapist immediately after each visit. In addition to the activities completed on the visits (visit content and ADL) information was recorded on the amount of administration and office based liaison time and travel time. The data were entered into a Microsoft Excel spreadsheet and analysed using Microsoft Excel and Stata (version 13) to produce summary statistics.

\section{Acceptability Questionnaire}

An acceptability questionnaire was designed by the research team. The design was informed based on a previous acceptability study (Fletcher-Smith, 2011) and was piloted with lay members of the research team. The questionnaire was sent by post to each participant who received the occupational therapy intervention after their discharge from the re-ablement service and at the end of the occupational therapy intervention. The questionnaire was designed to identify experiences of the intervention and asked the following questions:

1. Do you think the occupational therapist helped you to manage activities more easily? (Response options ranged from not at all to very much so) If yes, specify which particular tasks 
2. Do you think that the number of visits made by the occupational therapist was: too few, about right, too many?

3. Do you think that the time spent on each visit made by the occupational therapist was: not long enough, about right, too long?

4. Overall, how satisfied were you with the occupational therapy treatment you received? (Response options ranged from 'not at all satisfied' to 'extremely satisfied')

Open ended questions were included at the end to allow participants to comment freely (Kelley et al., 2003) on any aspect of the intervention. Questionnaires were accompanied by a letter and stamped return envelope from one author (MFW) who was not directly involved in delivering the intervention. If no reply was received within two weeks a second copy was mailed. No further contact attempts were made. Data from completed questionnaires were entered into a Microsoft Excel Spreadsheet. Data were analysed using Microsoft Excel and Stata to produce summary statistics.

\section{Key Informant Interviews}

Key informants are people who are identified because of their position and status within the population of interest (Gilchrist and Williams, 1999). The aim was to interview up to five key informants who had received the intervention. They were individuals who, from the occupational therapist's perspective, had received the intervention as planned and also those where there had been difficulties in delivering the intervention. The aim was to supplement and add depth to the findings from the acceptability questionnaire. The purpose of the interviews was to explore issues related to the acceptability of the intervention in greater detail than in the questionnaire.

Semi-structured interviews were selected in order to ensure that all relevant areas were covered and so that additional information could be volunteered from participants. A topic guide was developed and adapted from a previous study by Glendinning et al. (2010) in which re-ablement service users were interviewed. The topic guide is available by contacting the corresponding author by email ${ }^{1}$. In addition to general questions about the re-ablement service, particular emphasis was placed on the intervention provided by the occupational therapist, including:

- The particular ADL that the occupational therapist focussed on

- How goals were decided or agreed

\footnotetext{
1 phillip.whitehead@northumbria.ac.uk
} 
- Whether the occupational therapy input helped them to manage activities more easily

- In 'an ideal world' whether there was anything else that the occupational therapist could or should have done.

Interviews were completed by a research assistant who was not otherwise involved in the study or in delivering the intervention. Interviews were audio recorded, transcribed verbatim. They were analysed thematically using the six stage process outlined by Braun and Clarke (2006). An inductive data-led approach was used to carry out the analysis and the intention was to be led by the data with a focus on exploring aspects of the intervention which 'worked well' and where there had been difficulties. All recordings were listened to several times and each transcript was read several times. Initial notes were made as ideas emerged and particular sections of interest were highlighted for close attention and scrutiny. Data were then coded and all items that were relevant to the topic were given an initial code. Items were grouped together based on the initial codes to form themes. The themes were checked and cross-referenced to the initial coded extracts and then named. This process was conducted by the first author who was the occupational therapist who delivered the intervention.

\section{Results}

\section{Results: Content of the Occupational Therapy Intervention}

Thirteen participants $(87 \%)$ in the intervention group completed the re-ablement episode and their data were analysed. The median length of the re-ablement episode was 56 days, (range 20 to 126 days). Seventy-one visits were made to the participants by the occupational therapist. The median number of visits was 5 (range 2 to 13). The total time spent on all visits was 53 hours; therefore on average each participant received 4 hours occupational therapy contact time. The median visit length was 45 minutes (range 15 to 90). For every hour of contact time the occupational therapist spent an average of 45 minutes completing office-based tasks, including note writing and liaison with the wider re-ablement team and other health and social care professionals and ordering equipment and adaptations. Some of this liaison related to the research procedures and processes, in addition to the direct occupational therapy component. Each visit involved travel time of, on average 35 minutes (return journey). Overall, each participant received an average of 10 hours of occupational therapy time (4 hours direct contact, 3 hours administration and liaison, and 3 hours travel time).

A total of 28 goals were set for the 13 participants; the median number of goals set was 2 (range 1 to 4 ). Goals were categorised into groups based on ADL. The most common 
goal was in bathing/showering with eight participants (62\%) having a goal in this area. Six $(46 \%)$ had a goal in relation to kitchen activities, four $(31 \%)$ for strip washing, and three $(23 \%)$ for outdoor mobility. Figure 1 shows the total number of goals in each ADL category. In addition, two goals were set in relation to ongoing falls prevention, however, these could not be reviewed within the re-ablement episode as the outcome is a long-term goal. Sixteen goals (57\%) were fully achieved by the participants. Nine goals (32\%) were partially achieved. The main reasons why goals were partially achieved were that they concerned outdoor mobility and where the participant was not well enough to participate in this or weather was too inclement. Three goals (11\%) were not achieved. Two of these were in relation to the participants' deterioration in condition, such that they were not well enough to work on the goals. One was in relation to the participant moving to a different property and not being able to work on the goal which had originally been set.

[Insert Figure 1 here]

Figure 2 shows the overall content of the visits split into the percentage of the occupational therapist's time spent on particular activities: assessment, goal-setting, goal-reviewing, teaching techniques, practicing activities and general advice. The majority of time was spent on assessment (29\%), followed by case management and advice and support $(24 \%)$, and practicing activities (19\%). Considerable time was spent on initial assessment and all participants received this full initial assessment. Some had a low number of subsequent visits following the assessment which has increased the overall proportion of time spent on assessment. Time not allocated to individual categories was coded as general advice and case management, and this formed a large component of the intervention. Although the latter might seem lengthy it is consistent with other studies which have reported occupational therapy time use within interventional studies (Phillips et al., 2010; Sackley et al., 2015). Review of equipment and adaptations was coded as practising activities as this involved demonstration of use.

[Insert Figure 2 here]

Figure 3 shows the content of the visits categorised in respect of the various ADL activities that were worked on. These ADL categories are consistent with the goals that were set, with bathing and showering being the principal area followed by kitchen activities. 'Other' included categories of ADL that were too small to form standalone categories for example, transfers and pressure care etc.

[Insert Figure 3 here] 


\section{Results: Acceptability Questionnaire}

Questionnaires were returned from eight of the 13 participants who completed the intervention, a $62 \%$ response rate. The characteristics of those who returned the questionnaires compared to those who did not, are shown in Table 1. All respondents reported that the occupational therapist helped them to manage daily activities more easily: five (62\%) said 'somewhat' and three (38\%) said 'very much so'. When asked to comment on those activities that the occupational therapist helped them to manage, the following are examples of free text responses, again demonstrating the emphasis placed by the participants on bathing and showering:

- "Managing to shower myself independently" (Participant 08) Peer Review 10

- "Help with the shower with wall seat fitted and useful tips" (Participant 13)

All participants reported being satisfied with the occupational therapy intervention received: four (50\%) were 'satisfied' and four (50\%) were 'extremely satisfied'. Seven participants $(88 \%)$ reported that the number of visits made by the occupational therapist was 'about right'. One reported that the number of visits was 'too few'; this participant also took part in a qualitative interview in which she clarified that she would have liked the occupational therapy treatment to continue after the re-ablement service had ended. No participants reported there were too many visits. All respondents said that time spent on each visit was 'about right'. Participants were asked to describe, in free text, "the most helpful things that the occupational therapist did". The responses were divided between items that were provided (stairlift referral and long-handled sponge) and "advice", "talking" and "reassurance". On the whole, the results from the acceptability questionnaire suggest that the occupational therapy intervention was acceptable and that the respondents believed it helped them to carry out activities independently, with particular reference to personal care activities.

[Insert Table 1 here]

\section{Results: Key Informant Interviews}

Five participants from the intervention group were interviewed and their characteristics are shown in Table 1. Two interview participants had poor memories so they had difficulty recalling specific information as to what happened during the intervention period, therefore the data collected from these interviewees was limited. The interviews ranged in duration from 18 to 49 minutes with a mean of 32 (SD 12) minutes. The findings have been categorised into four themes: Experiencing Homecare Re-ablement; Difficulties 
Encountered with Homecare Re-ablement; Boosting Confidence and Ability to Cope; and Extending Homecare Re-ablement Beyond Personal ADL.

\section{Theme 1: Experiencing Homecare Reablement}

The first theme focussed on the activities that the occupational therapist and re-ablement team assisted with, within a programme which was gradually reduced. Two participants described a programme whereby they were supported to regain independence with showering and kitchen activities and a third participant (with poor memory) partially described a graded dressing intervention. The quotation below illustrates the graded ADL programme that was described for ADL within the home:

\footnotetext{
"[the OT] came and got me a seat put in [the shower]... I had the carers come just to watch me at first, you see, to see how I went on. But.. er, it come to a point where I didn't really need them, so.. er, I manage it alright now" Intervention Participant 013.
}

\section{Theme 2: Difficulties Encountered with Homecare Re-ablement}

Some participants within the intervention group as a whole were too unwell to participate fully in the occupational therapy and re-ablement programme. One participant described being unable to participate due to fatigue and weakness as the quotation below demonstrates:

"I was so tired at the time, I didn't work" Intervention Participant 026

Another participant described having difficulties as her property was not suited to her needs. This led to her being at a "very low ebb" such that it was not possible to work on re-ablement goals during much of the episode:

"Yeah I was at a very low ebb, not crying all over the place but you know really low, and I'd got to the point where I thought 'this is it, I'm not bothered, there's not much going off here'"I Intervention Participant 016

Both of these participants had difficulty in participating and did not describe a graded programme to the same extent as the others. Outdoor mobility was a particular component of the intervention where delivery was problematic. One participant described walking outdoors for the first time in two years, with the occupational therapist, but went on to describe how her condition deteriorated such that she struggled to maintain the progress: 
"[the OT] come and took me a walk round... We only went 'round the block, but, erm, you know, I haven't managed it again on my own, I keep thinking I will do but as I say my arthritis has got worse." Intervention Participant 013

\section{Theme 3: Boosting Confidence and Ability to Cope}

All five participants spoke about the support, advice and encouragement received by the occupational therapist as being valuable and assisting to boost their confidence to manage activities and/or to cope at home. The "mental support" and encouraging approach were appreciated and were believed to be key facilitators. In these interviews all the key-informants spoke about the advice, support and encouragement that they received from the occupational therapist as being beneficial to them:

"I can remember [the OT] explaining and telling me things... at the time it helped me, it helped me... His attitude was that if I wanted to, I could... do, the thing I wanted to. He encouraged me..." Intervention Participant 030

\section{Theme Four: Beyond Personal ADL}

In response to the question: "In an ideal world is there anything else the occupational therapist could have done" four of the participants stated that they could not think of any additional actions that would have been needed. They indicated that the time limit of the intervention was appropriate in that they were able to manage independently by the end of the re-ablement episode such that further input was not needed or that their aspiration for further achievements were limited. However, Participant 016, discharged with an ongoing homecare service, stated that continued, ongoing support from the occupational therapist would have been valuable beyond the end of the intervention period. This was in order to respond to her changing needs and situation, as the quotation below illustrates:

\footnotetext{
"Whereas I think with occupational therapy... it could be more valuable than what people or what the authorities or what health people think. I think it could be a lot more valuable to people like myself if we saw OT not just for a certain period of time but... Ongoing...My needs might be different. I might need different equipment, I don't know what I might need" Intervention Participant 016
}

\section{Discussion and Implications}

The principal strength of this study is that, to our knowledge, no previous studies have described or evaluated occupational therapy involvement within homecare reablement services. The results from the OTHERS feasibility RCT suggested that there were positive 
trends associated with the occupational therapy intervention compared with the control group (Whitehead et al., 2016). This paper has provided additional detail on the content of the occupational therapy intervention and evaluated participants views and experiences of it.

The main activities prioritised by participants were personal ADL (bathing, showering and stripwashing) and kitchen activities. The focus on these activities worked particularly well and participants reported that they valued the combination of equipment/adaptation provision, the graded approach, and the "mental support" which facilitated confidence boosting and their ability to cope. It is possible that these activities were prioritised by participants as these were inextricably linked with the need for an ongoing homecare service, i.e. these were the activities that that homecare workers would have provided on a continuing basis after the re-ablement episode had ended. In order to resume independent living participants had to regain independence with ADL within the home. By the time these goals had been achieved, the re-ablement service was coming to an end which meant that there was insufficient time to focus on additional or extended ADL goals

Although on the whole the intervention worked well and was acceptable, there were aspects which were difficult. Participants' fluctuating physical and mental health affected their ability to participate, particularly following the initial event which had led to the need for the service. Furthermore, three participants had an outdoor mobility goal, emphasising the importance of outdoor mobility and community access to the participants. However, there were difficulties in achieving these goals with all participants due to fluctuations in their health conditions or inclement weather. Although they prioritised ADL within the home, they were left with ongoing, concurrent goals in relation to ADL outside the home which could not be addressed within the timescale of the re-ablement episode.

Findings from the interviews suggested that a further review or reviews, by an occupational therapist, after people have been discharged from the re-ablement service might assist with the maintenance of people's independence at home and promote further progress. The recent NICE guideline for Intermediate Care including re-ablement has made a research recommendation for further studies to evaluate the effectiveness of reablement beyond six weeks (National Institute for Health and Care Excellence, 2017) and this is consistent with the findings from the interviews. The NICE guideline also states that "Social and leisure goals should be legitimate goals of an Intermediate Care [including reablement] service". However, this study identified practical issues in delivering such goals within the context of a short-term re-ablement episode which was tied to a homecare service in which there was a prevailing focus on activities of daily living within the home. 
Notwithstanding the clear and obvious compatibilities between the tenets of occupational therapy practice and re-ablement services there is limited empirical research on occupational therapy in re-ablement. This study has provided additional detailed information on the content of an occupational therapy intervention and the perspectives of the people who received it. It highlights that although occupational therapists and reablement service users may consider wider holistic goals and needs, there are often practical considerations and time constraints that affect what can be achieved within the confines of a re-ablement episode.

There are some limitations with this study. The majority of data collection and analysis was completed by one occupational therapist who delivered the intervention in the RCT. This means that there was a possibility for bias. However this was minimised, where possible, by having another researcher conduct the interviews and receive the returned questionnaires (i.e. not the treating occupational therapist directly). Although the findings from the acceptability of the intervention were unequivocally positive they were based on $62 \%$ of intervention participants and the sample was small. Whilst there is no reason to indicate that these respondents were not representative of the intervention participant group, it is important to note that the data do not represent the complete sample.

\section{Conclusion}

We found that it was possible to deliver a graded ADL programme within the home, particularly focusing on personal care and kitchen activities, for the majority of participants in a way that was acceptable to them. Participants valued the intervention and appreciated the support, advice and confidence building provided by the occupational therapist. There were, however, some difficulties in delivering the intervention as planned, which were primarily due to the vulnerable and fragile nature of the group. Where participants had goals in relation to activities outside the home it was difficult to incorporate these within the timescales of the re-ablement service. Occupational therapists should also consider research on outdoor mobility and wider community participation as part of re-ablement services; this would be consistent with the wishes and person-centred goals of the participants in this study. It might be pertinent to focus on extended ADL including social, leisure and outdoor mobility, at a later stage; further research should explore this. 


\section{Key findings}

- An intervention focussing on ADL within the home was acceptable, although some participants also had extended ADL goals which could not be achieved in the timescale

- Participants particularly valued the advice, support and reassurance provided by the occupational therapist

- Further research focussing beyond ADL within the home and a 6-week time period is warranted

\section{What the study has added}

This study has provided a detailed description of the content and analysis of the acceptability of an occupational therapy intervention in homecare re-ablement. Whilst the intervention was acceptable to participants, an additional focus beyond 6-weeks and on activities outside the home is warranted.

\section{Acknowledgements}

The authors would like to thank the following for their assistance in conducting this research. The project steering group: Professor Jill Manthorpe (chair), Stuart Belshaw, Dr Adam Gordon, Zaid Latif, Ian McGeorge, Joanne Martinsons and Rachel Mellor. The participants and their families. Nottingham City Council and particularly the occupational therapy and Intake Re-ablement teams. Dr Clare Mann for her assistance with the data collection.

\section{Research Ethics}

Ethical approval was obtained from the Social Care Research Ethics Committee (Ref: 13/IEC08/002). All participants provided written informed consent to be interviewed for and have their anonymised data used in the research study.

\section{Declaration of Conflicting Interests}

The authors declared no potential conflicts of interests with respect to the research, authorship, and/or publication of this article.

\section{Funding}

This article is independent research supported by the National Institute for Health Research (Doctoral Research Fellowship, DRF-2012-05-131). The views expressed are those of the authors and not necessarily those of the NHS, the National Institute for Health Research or the Department of Health. 


\section{References}

Allen K and Glasby J. (2013) 'The Billion Dollar Question': Embedding Prevention in Older People's Services - Ten 'High-Impact' Changes. British Journal of Social Work 43(5): 904924.

Aspinal F, Glasby J, Rostgaard T, et al. (2016) New horizons: Reablement - supporting older people towards independence. Age and Ageing 45(5): 574-578.

Braun V and Clarke V. (2006) Using thematic analysis in psychology. Qualitative Research in Psychology 3(2): 77-101.

College of Occupational Therapists. (2010) Reablement: the added value of Occupational Therapists. London: College of Occupational Therapists.

Craig P, Dieppe P, Macintyre S, et al. (2013) Developing and evaluating complex interventions: The new Medical Research Council guidance. International Journal of Nursing Studies 50(5): 587-592.

Department of Health. (2017) Statutory guidance to support local authorities to implement the Care Act. London: Department of Health.

Drummond A. (2017) Feasibility and pilot studies: Why are they important? British Journal of Occupational Therapy 80: 335-336.

Fletcher-Smith J. (2011) Recovery of dressing ability after stroke. MPhil Thesis. Division of Rehabilitation and Ageing. University of Nottingham.

Gilchrist V and Williams R. (1999) Key Informant Interviews. In: Crabtree B and Miller W (eds) Doing Qualitative Research. 2nd ed. Thousand Oaks, California.: Sage Publications, 71-88.

Glendinning C, Jones K, Baxter K, et al. (2010) Home Care Re-ablement Services: Investigating the Longer Term Impacts (Prospective Longitudinal Study), York: Social Policy Research Unit, University of York.

Grant M, Radford K, Sinclair E, et al. (2014) Return to work after stroke: recording, measuring, and describing occupational therapy intervention. British Journal of Occupational Therapy 77(9): 457-465. 
Hasson H, Blomberg S and Dunér A. (2012) Fidelity and moderating factors in complex interventions: a case study of a continuum of care program for frail elderly people in health and social care. Implementation Science 7(1): 1-11.

Hoffmann TC, Glasziou PP, Boutron I, et al. (2014) Better reporting of interventions: template for intervention description and replication (TIDieR) checklist and guide. BMJ,348, 1687

Kelley K, Clark B, Brown V, et al. (2003) Good practice in the conduct and reporting of survey research. International Journal for Quality in Health Care 15(3): 261-266.

Le Mesurier N and Cumella S. (1999) Enhancing Independence: The Effectiveness of ReAblement Provision in South Worcestershire. Managing Community Care 7(4): 27- 32.

National Institute for Health and Care Excellence. (2017) Intermediate Care including reablement [NICE guideline NG74]. London: National Institute for Health and Care Excellence.

NHS England. (2014) National Audit of Intermediate Care: Summary Report. London: NHS England.

Pettersson C and Iwarsson S. (2017) Evidence-based interventions involving occupational therapists are needed in re-ablement for older community-living people: A systematic review. British Journal of Occupational Therapy 80(5): 273- 285.

Phillips J, Drummond A, Radford K, et al. (2010) Return to Work after Traumatic Brain Injury: Recording, Measuring and Describing Occupational Therapy Intervention. British Journal of Occupational Therapy 73(9): 422-430.

Sackley CM, Walker MF, Burton CR, et al. (2015) An occupational therapy intervention for residents with stroke related disabilities in UK care homes (OTCH): cluster randomised controlled trial. BMJ 350: h468.

Social Care Institute for Excellence. (2011) Reablement: a key role for occupational therapists, London: Social Care Institute for Excellence. 
Walker MF, Hoffmann TC, Brady MC, et al. (2017) Improving the development, monitoring and reporting of stroke rehabilitation research: Consensus-based core recommendations from the Stroke Recovery and Rehabilitation Roundtable. International Journal of Stroke 12(5): 472-479.

Whitehead P. (2016) Can an occupational therapy intervention increase independence in activities of daily living ( $A D L$ ) in people who use homecare re-ablement services? PhD Thesis, School of Health Sciences. University of Nottingham, UK.

Whitehead PJ, Drummond AE, Walker MF, et al. (2014) Occupational Therapy in HomEcare Re-ablement Services (OTHERS): study protocol for a randomized controlled trial. Trials 15(1): 447.

Whitehead PJ, Walker MF, Parry RH, et al. (2016) Occupational Therapy in HomEcare Reablement Services (OTHERS): results of a feasibility randomised controlled trial. BMJ Open 6: e011868.

Whitehead PJ, Worthington EJ, Parry RH, et al. (2015) Interventions to reduce dependency in personal activities of daily living in community dwelling adults who use homecare services: A systematic review. Clinical Rehabilitation 29(11): 1064- 1076.

Wilkins S, Jung B, Wishart L, et al. (2003) The Effectiveness of Community-Based Occupational Therapy Education and Functional Training Programs for Older Adults: A Critical Literature Review. Canadian Journal of Occupational Therapy 70(4): 214-225. 
Figure 1: Goals by ADL Category

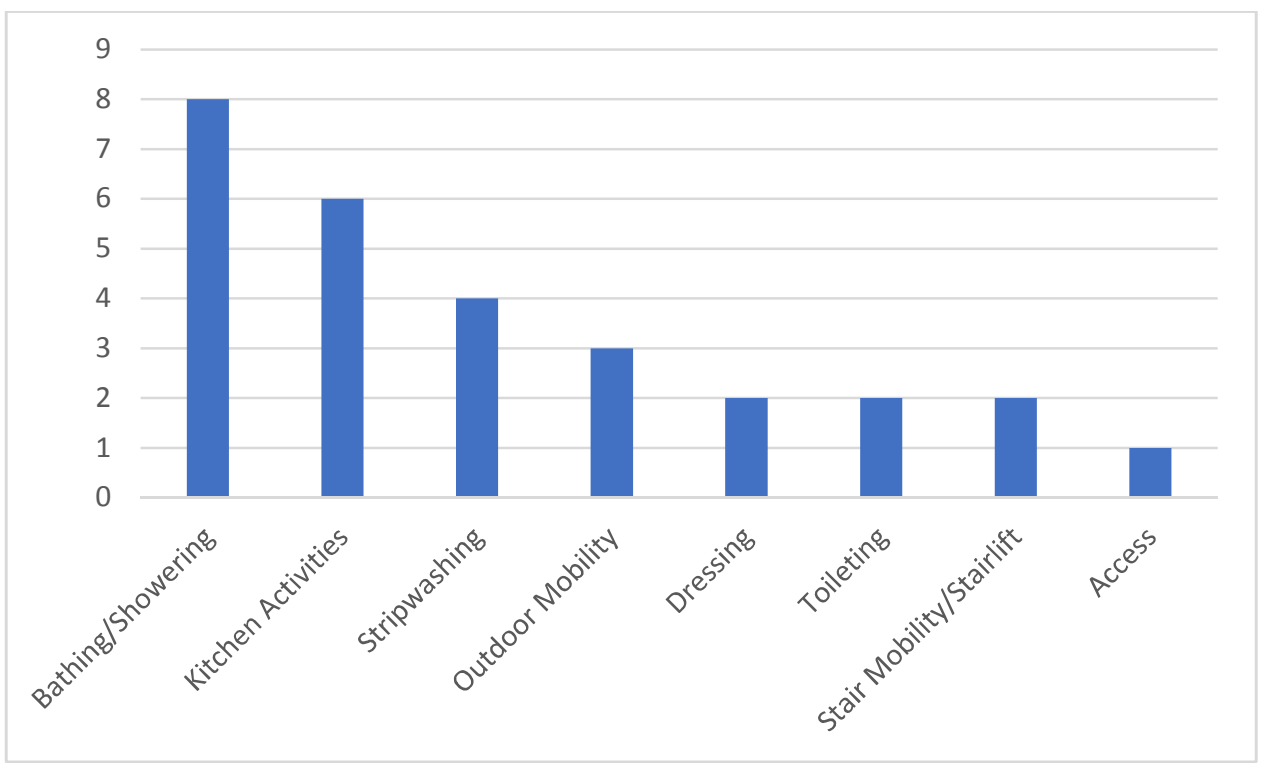


Figure 2: Visit Content - Overall

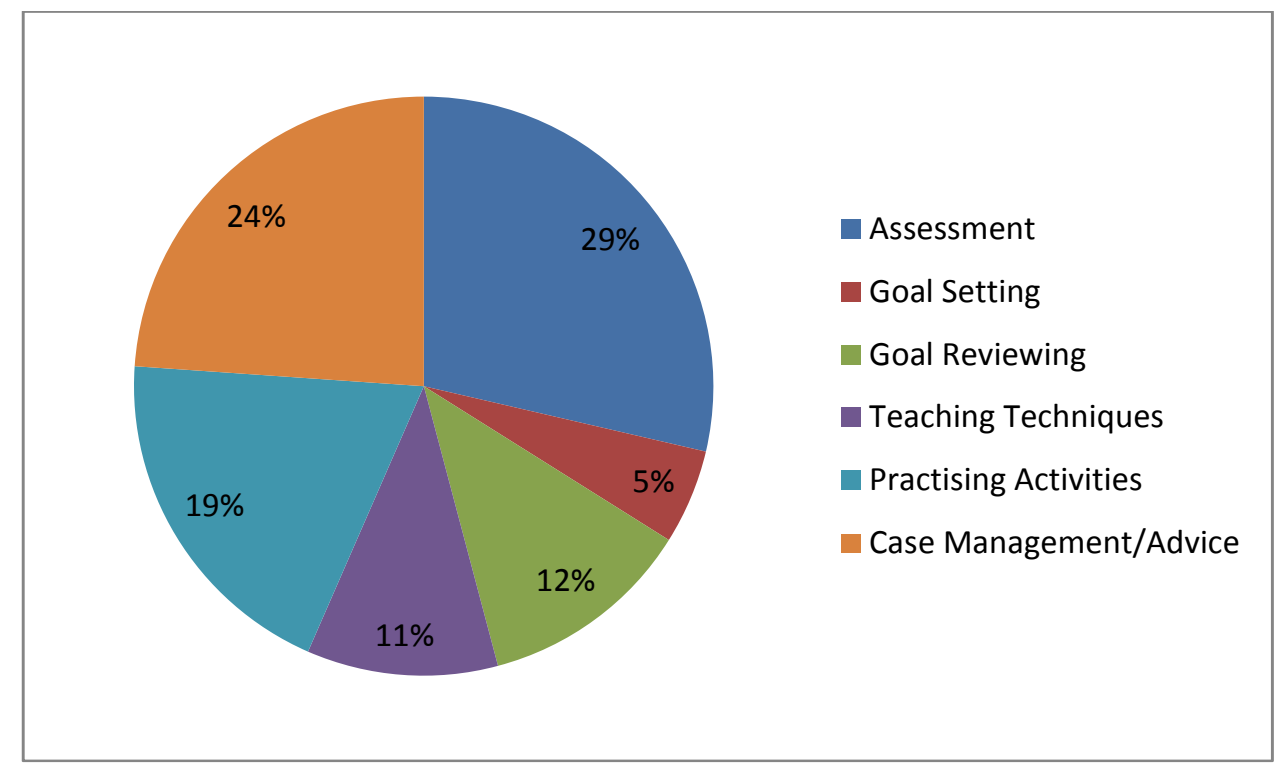


Figure 3: Visit Content - Activities of Daily Living

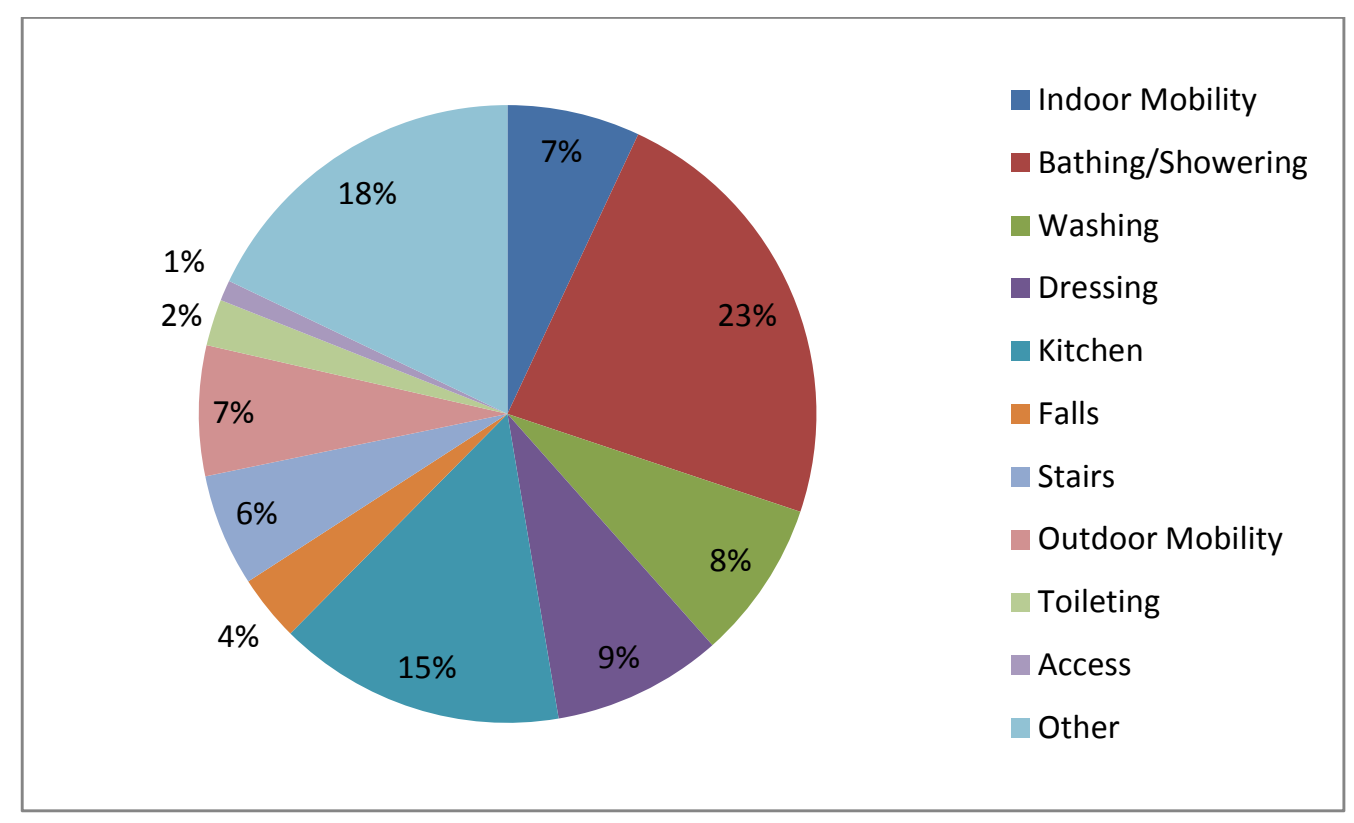


Table 1: Characteristics of Questionnaire Responders and Interviewees

\begin{tabular}{|l|c|c|}
\hline & \multicolumn{2}{|c|}{ Questionnaires } \\
\hline $\begin{array}{c}\text { Returned } \\
\text { Questionnaire } \\
(\mathbf{n = 8})\end{array}$ & $\begin{array}{c}\text { Did not Return } \\
\text { Questionnaire } \\
(\mathbf{n = 5})\end{array}$ \\
\hline $\begin{array}{l}\text { Gender } \\
\text { Age }\end{array}$ & $2(20 \%)$ & $2(40 \%)$ \\
\hline $\begin{array}{l}\text { Lives Alone } \\
\text { Yes }\end{array}$ & $83(\mathrm{SD} 8.9)$ & $80.4(\mathrm{SD} 10.1)$ \\
\hline $\begin{array}{l}\text { Ethnicity } \\
\text { White British } \\
\text { Other }\end{array}$ & $6(75 \%)$ & $2(40 \%)$ \\
\hline & $7(88 \%)$ & $3(60 \%)$ \\
\hline & $1(12 \%)$ & $2(40 \%)$ \\
\hline $\begin{array}{l}\text { Gender } \\
\text { Male }\end{array}$ & $\begin{array}{c}\text { Interviewed } \\
(\boldsymbol{n = 5})\end{array}$ & $\begin{array}{c}\text { Not Interviewed } \\
(\boldsymbol{n}=\mathbf{8})\end{array}$ \\
\hline Age & $1(20 \%)$ & $3(38 \%)$ \\
\hline $\begin{array}{l}\text { Lives Alone } \\
\text { Yes }\end{array}$ & $82.4(\mathrm{SD} 9.86)$ & $81.8(\mathrm{SD} 9.7)$ \\
\hline $\begin{array}{l}\text { Ethnicity } \\
\text { White British } \\
\text { Other }\end{array}$ & $2(40 \%)$ & $6(75 \%)$ \\
\hline
\end{tabular}

